\title{
CONHECIMENTO DO ENFERMEIRO SOBRE A PREVENÇÃO DO PÉ DIABÉTICO: REVISÃO INTEGRATIVA DA LITERATURA
}

\section{Nurses' knowledge about the prevention of the diabetic foot: an integrative literature review \\ Conocimiento del enfermero sobre la prevención del pie diabético: revisión integrativa de la literatura}

Luana Savana Nascimento de Sousa

Universidade Federal do Piauí (UFPI) - Teresina (PI) - Brasil

Malvina Thaís Pacheco Rodrigues

Universidade Federal do Piauí (UFPI) - Teresina (PI) - Brasil

Márcio Dênis Medeiros Mascarenhas

Universidade Federal do Piauí (UFPI) - Teresina (PI) - Brasil

Ana Roberta Vilarouca da Silva

Universidade Federal do Piauí (UFPI) - Teresina (PI) - Brasil

\section{RESUMO}

Objetivo: Sistematizar o conhecimento do enfermeiro sobre a prevenção do pé diabético. Métodos: Trata-se de uma revisão integrativa da literatura, realizada no período de abril a junho de 2016, nas bases de dados: SciELO, LILACS e PubMed, com os seguintes descritores: Conhecimento; Enfermagem; Prevenção primária; Pé diabético (Knowledge; Nursing; Primary Prevention; Diabetic foot). Resultados: Foram selecionados sete artigos, sendo evidenciado que o conhecimento do enfermeiro está pautado em ações de orientação e cuidado na prevenção do pé diabético, com enfoque importante na higienização e cuidado com unhas, calos e calçados. O instrumento de avaliação dos pés mais utilizado foi o Monofilamento de $10 \mathrm{~g}$. Conclusão: Conclui-se que os enfermeiros estão em contato diário com os pacientes diabéticos, mas que as ações muitas vezes estão direcionadas para atividades exclusivas de orientação quanto à doença em si, e, como consequência, o conhecimento acerca das orientações determinadas pelas diretrizes para prevenir o pé diabético torna-se insuficiente.

Descritores: Conhecimento; Enfermagem; Prevenção primária; Pé diabético.

\section{ABSTRACT}

Objective: To systematize nurses' knowledge about the prevention of the diabetic foot. Methods: Integrative literature review carried out from April to June 2016 in SciELO, LILACS and PubMed databases using the following descriptors: Knowledge; Nursing; Primary prevention; Diabetic foot. Results: Seven articles were selected, and they showed that the nurse's knowledge is based on counseling and care actions aimed at the prevention of the diabetic foot with a special focus on nail, calluses and shoes hygiene and care. The most used instrument for examining the feet was the $10 \mathrm{~g}$ Monofilament. Conclusion: The nurses have contact with diabetic patients every day, but their actions often include activities exclusively aimed at disease counseling; therefore, their knowledge about the guidelines for preventing the diabetic foot is poor.

Descriptors: Knowledge; Nursing; Primary Prevention; Diabetic foot. 


\section{RESUMEN}

Objetivo: Sistematizar el conocimiento del enfermero sobre la prevención del pie diabético. Métodos: Se trata de una revisión integrativa de la literatura realizada entre abril y junio de 2016 en las bases de datos SciELO, LILACS y PubMed con los siguientes descriptores: Conocimiento; Enfermería; Prevención Primaria; Pie Diabético (Knowledge; Nursing; Primary Prevention; Diabetic foot). Resultados: Siete artículos fueron elegidos y ha sido evidenciado que el conocimiento del enfermero está fundamentado en acciones de orientación y cuidado para la prevención del pie diabético con importancia para la higiene y cuidado de las uñas, callos y calzados. El instrumento más utilizado para la evaluación de los pies ha sido el Monofilamento de $10 \mathrm{~g}$. Conclusión: Se concluye que los enfermeros tienen contacto a diario con pacientes diabéticos pero las acciones se concentran muchas veces en actividades exclusivas de orientación sobre la enfermedad $y$, en consecuencia, el conocimiento sobre las orientaciones que son determinadas por las directrices para la prevención del pie diabético es insuficiente.

Descriptores: Conocimiento; Enfermería; Prevención Primaria; Pie Diabético.

\section{INTRODUÇÃO}

A diabetes mellitus (DM) é uma das doenças crônicas mais prevalentes em nível global, apresentando forte impacto na morbidade dos indivíduos, que é decorrente de complicações agudas e crônicas e da alta taxa de hospitalizações e de mortalidade, gerando significativos danos econômicos e sociais ${ }^{(1,2)}$.

O pé diabético está entre as complicações mais frequentes da DM e suas consequências podem ser traumáticas à vida do indivíduo, pois envolve desde feridas crônicas até amputações de membros inferiores. O exame periódico dos pés propicia a identificação precoce e o tratamento oportuno das alterações encontradas, possibilitando a prevenção de um número expressivo de complicações do pé diabético ${ }^{(3)}$.

O enfermeiro tem papel indispensável na avaliação sistemática dos pés e na identificação precoce dos fatores de risco, proporcionando a redução de úlcera e amputações. Essa avaliação deverá ser associada à história clínica do paciente, investigando a ocorrência de lesões ou amputações prévias, e à observação se há incapacidade do paciente para realizar o autocuidado com os pés ${ }^{(4)}$.

A investigação científica acerca do conhecimento do enfermeiro quanto à prevenção do pé diabético é de grande importância, porque permite identificar a qualidade da assistência prestada, além de proporcionar a reformulação de estratégias para evitar complicações por DM. Diante disso, o estudo teve como objetivo sistematizar o conhecimento do enfermeiro sobre a prevenção do pé diabético.

\section{MÉTODOS}

Trata-se de uma revisão integrativa da literatura, que se caracteriza por permitir a busca, a avaliação crítica e a síntese de evidências disponíveis sobre o tema investigado, sendo o seu produto final o estado atual do conhecimento do tópico de interesse, bem como a identificação de lacunas que direcionam para o desenvolvimento de futuras pesquisas ${ }^{(5)}$.

A revisão envolveu as seguintes etapas: identificação do tema e seleção da hipótese, ou da questão de pesquisa, para elaboração da revisão; estabelecimento de critérios para inclusão e exclusão de estudos/amostragem, assim como para busca na literatura; definição das informações a serem extraídas dos estudos selecionados/categorização dos estudos; avaliação dos estudos incluídos na revisão; interpretação dos resultados; apresentação da revisão/síntese do conhecimento ${ }^{(6)}$.

O estudo ocorreu no período de abril a junho de 2016 e foram consultadas as bases de dados eletrônicas: Scientific Electronic Library on Line (SciELO), Literatura Latino Americana e do Caribe em Ciências da Saúde (LILACS) e United States National Library of Medicine/ Medical Literature Analysis and Retrieval System on-line (PubMed/Medline), disponíveis na Biblioteca Virtual em Saúde (BVS). Utilizou-se o DeCS (Descritores em Ciências da Saúde) e o MeSH (Medical Subject Headings) para a verificação dos descritores, os quais consistiram nos seguintes: Knowledge; Nursing; Primary Prevention; Diabetic foot. A busca foi realizada utilizando descritores em inglês e associando-os ao conectivo booleano "and".

Para organização e síntese dos achados, a pré-seleção dos artigos foi realizada mediante a leitura dos respectivos títulos e resumos, a fim de verificar a adequação aos critérios de inclusão e exclusão. Como critérios de inclusão, consideraram-se os seguintes: textos disponíveis on-line na íntegra; nos idiomas português e inglês; que abordassem a temática em questão; publicados entre os anos de 2011 e 2016. Os critérios de exclusão compreenderam: os artigos duplicados nas bases de dados, as teses e os documentos institucionais. A seleção dos artigos a serem incluídos no estudo foi realizada por duas pessoas e de forma independente para garantir a elegibilidade da pesquisa. 
Os artigos selecionados foram descritos e caracterizados de acordo com o título, nomes dos autores, periódico de publicação, ano de publicação, base de dados, objetivos, metodologia empregada e principais desfechos para a temática, sintetizando os resultados por similaridade do conteúdo.

Realizou-se a interpretação dos resultados por meio da análise crítica dos estudos revisados, a qual proporcionou a investigação do conhecimento teórico/prático do enfermeiro na prevenção do pé diabético, com enfoque nas ações (orientações e cuidados), que são as implicações resultantes desta revisão integrativa, além de possibilitar a identificação das principais estratégias e dificuldades encontradas para desenvolver as práticas preventivas.

\section{RESULTADOS}

Para a revisão integrativa, foram encontradas 465 potenciais referências, das quais somente 07 compreenderam aos critérios de inclusão e exclusão preestabelecidos. Os dados descritos na figura 1 representam a seleção da amostra nas três bases de dados escolhidas para o estudo.

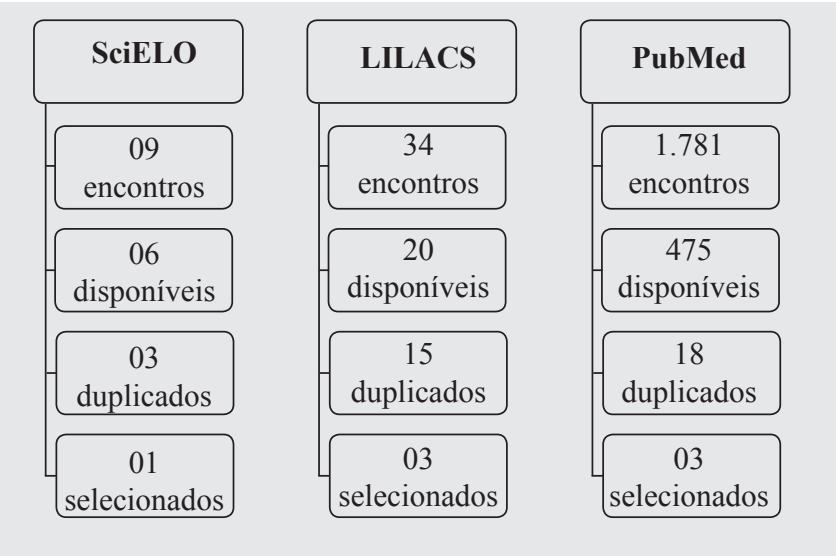

Figura 1 - Fluxograma do processo de identificação e seleção dos artigos segundo base de dados.

No que se refere ao perfil dos artigos encontrados, verificou-se predomínio das bases de dados LILACS e PubMed; de estudos com abordagem quantitativa e de estudos do tipo transversal, tendo como principais instrumento de avaliação a aplicação de questionários estruturados, semi-estruturados e entrevistas. Adicionalmente, observou-se que a maioria das pesquisas se concentrou em periódicos da área de enfermagem e saúde pública, e buscavam conhecer a atitude, o conhecimento e a prática do enfermeiro na prevenção do pé diabético, investigando a identificação das lesões nos pés, os cuidados e as orientações quanto à prevenção do pé diabético (Quadro I).

Quadro I - Caracterização dos artigos segundo autor, ano, local, conhecimentos, estratégias e dificuldades.

\begin{tabular}{|c|c|c|c|c|c|}
\hline Autor & Ano & Local & $\begin{array}{c}\text { Conhecimento } \\
\text { (orientações/cuidados de } \\
\text { prevenção) }\end{array}$ & Estratégias & Dificuldades \\
\hline Cubas et $\mathrm{al}^{(7)}$ & 2013 & $\begin{array}{l}\text { Curitiba } \\
\text { (PR) }\end{array}$ & $\begin{array}{l}\text { Orientações quanto ao uso de } \\
\text { calçados e corte das unhas; } \\
\text { Tipos de meias; } \\
\text { Avaliação da sensibilidade } \\
\text { protetora e tátil, utilizando o } \\
\text { estesiômetro (monofilamento } \\
\text { de Semmes-Wertein). }\end{array}$ & $\begin{array}{l}\text { Ação multiprofissional } \\
\text { para potencializar as } \\
\text { orientações e aumentar } \\
\text { e aumentar a aderência } \\
\text { das mesmas; } \\
\text { Atividades educativas. }\end{array}$ & $\begin{array}{l}\text { Falta de orientações } \\
\text { importantes; }\end{array}$ \\
\hline
\end{tabular}




\begin{tabular}{|c|c|c|c|c|c|}
\hline Aalaa et al ${ }^{(8)}$ & 2012 & Teerã (Irã) & $\begin{array}{l}\text { Orientações quanto à limpeza } \\
\text { dos pés, cuidado com a pele a } \\
\text { as unhas; } \\
\text { Orientações quanto a } \\
\text { hidratação, utilização de } \\
\text { meias e sapato adequado; } \\
\text { Realização do teste } \\
\text { de sensibilidade } \\
\text { (monofilamento de Semmes- } \\
\text { Wertein) }\end{array}$ & $\begin{array}{l}\text { Treinamento especial } \\
\text { dos enfermeiros, para } \\
\text { o cuidado com os } \\
\text { pés, a fim de fornecer } \\
\text { serviços eficazes para } \\
\text { facilitar na promoção } \\
\text { da saúde dos pacientes } \\
\text { diabéticos; } \\
\text { Educação em saúde } \\
\text { pública. }\end{array}$ & $\begin{array}{l}\text { Formação acadêmica } \\
\text { dos enfermeiros, } \\
\text { insatisfatória; } \\
\text { Falta de enfermeiro } \\
\text { especialista na área. }\end{array}$ \\
\hline $\begin{array}{l}\text { Policarpo et } \\
\qquad \mathrm{al}^{(9)}\end{array}$ & 2014 & Picos (PI) & $\begin{array}{l}\text { Orientações de higienização } \\
\text { dos pés; } \\
\text { Cuidados com as unhas e } \\
\text { calos; } \\
\begin{array}{l}\text { Realização do teste de } \\
\text { sensibilidade. }\end{array}\end{array}$ & $\begin{array}{l}\text { Medidas educativas } \\
\text { (cartilhas, encontros } \\
\text { e visitas domiciliares) } \\
\text { voltadas à promoção } \\
\text { de conhecimentos } \\
\text { dessa clientela. }\end{array}$ & Falta de conhecimento. \\
\hline $\begin{array}{c}\text { Varaei et } \\
\mathrm{al}^{(10)}\end{array}$ & 2013 & Irã & $\begin{array}{l}\text { Orientações baseadas nas } \\
\text { evidências de enfermagem. }\end{array}$ & $\begin{array}{l}\text { Educação que } \\
\text { pode melhorar o } \\
\text { conhecimento e atitude } \\
\text { do enfermeiro. }\end{array}$ & $\begin{array}{lcc}\text { Práticas } & \text { de } & \text { saúde, } \\
\text { repassadas } & \text { de } & \text { forma } \\
\text { incorreta. } & & \end{array}$ \\
\hline $\begin{array}{l}\text { Pereira et } \\
\mathrm{al}^{(16)}\end{array}$ & 2013 & $\begin{array}{l}\text { Fortaleza } \\
\qquad(\mathrm{CE})\end{array}$ & $\begin{array}{l}\text { Orientações quanto à } \\
\text { higienização, hidratação (da } \\
\text { pele diariamente) e lixação } \\
\text { das unhas, ao invés de cortá- } \\
\text { las; } \\
\text { Inspeção estática do exame } \\
\text { físico; } \\
\text { Orientações para não andar } \\
\text { descalço. }\end{array}$ & $\begin{array}{l}\text { Promoção de educação } \\
\text { em saúde às pessoas } \\
\text { com diabetes mellitus. }\end{array}$ & $\begin{array}{l}\text { A anamnese e o exame } \\
\text { físico dos pés ocorreram } \\
\text { de modo incompleto, } \\
\text { deixando de cumpri } \\
\text { etapas importantes } \\
\text { na prevenção ao } \\
\text { desenvolvimento do } \\
\text { pé diabético ou das } \\
\text { potenciais complicações a } \\
\text { ele associadas; } \\
\text { Falta de orientações } \\
\text { importantes. }\end{array}$ \\
\hline Silva et $\mathrm{al}^{(18)}$ & 2012 & $\begin{array}{l}\text { Ponta } \\
\text { Grossa } \\
\text { (PR) }\end{array}$ & $\begin{array}{l}\text { Cuidados com os pés e a } \\
\text { pele. }\end{array}$ & $\begin{array}{l}\text { Atividades educativas } \\
\text { que visem o bem-estar } \\
\text { pessoal e social do } \\
\text { paciente. }\end{array}$ & Falta de conhecimento. \\
\hline Couto et $\mathrm{al}^{(24)}$ & 2014 & $\begin{array}{l}\text { Itabuna } \\
\text { (BA) }\end{array}$ & $\begin{array}{l}\text { Orientações referentes ao uso } \\
\text { de medicação adequada, dieta } \\
\text { alimentar, atividade física } \\
\text { regular, controle glicêmico e } \\
\text { necessidade de autocuidado } \\
\text { (higiene dos pés); } \\
\text { Utilização do monofilamento } \\
\text { de Semmes-Wertein. }\end{array}$ & $\begin{array}{l}\text { I } \mathrm{m} \text { p } 1 \text { e } \mathrm{m} \text { e } \mathrm{n} \mathrm{t} \text { a } \mathrm{r} \\
\text { medidas (estratégia } \\
\text { ou programas) que } \\
\text { proporcionem a } \quad \text { a } \\
\text { melhoria da assistência } \\
\mathrm{e} \text { a prevenção } \\
\text { de complicações } \\
\text { decorrentes da } \\
\text { neuropatia diabética. }\end{array}$ & $\begin{array}{l}\text { Falta de recursos } \\
\text { materiais; } \\
\text { Questão social que } \\
\text { dificulta a implementação } \\
\text { de algumas ações (falta } \\
\text { de saneamento básico } \\
\text { e a irregularidade do } \\
\text { abastecimento de água } \\
\text { nos bairros, o que dificulta } \\
\text { a higienização pessoal } \\
\text { e do lar, principalmente } \\
\text { de usuários com úlceras } \\
\text { extensas). }\end{array}$ \\
\hline
\end{tabular}


As informações repassadas para os usuários abordavam as seguintes ações: higienização dos pés; cuidados com as unhas (corte e lixamento) e calos (remoção); hidratação da pele; utilização de calçados adequados; importância da dieta alimentar (restrita) e atividade física, e o controle glicêmico, com o propósito de educar para o autocuidado e evitar as complicações do pé diabético.

Na investigação, quanto à utilização de instrumentos para a avaliação do pé, foram usados os seguintes instrumentos: Monofilamento de $10 \mathrm{~g}$ (5,07U) de Semmes-Weinstein, para sensibilidade tátil e protetora; Diapasão $128 \mathrm{H}$, para sensibilidade vibratória; e o Martelo neurológico, para o reflexo de Aquileu. Desses, o Monofilamento de Semmes-Weinstein foi o mais utilizado pelos profissionais para o exame físico.

Como estratégias para prevenção do pé diabético, destacaram-se as atividades de educação em saúde e visitas domiciliares, além do autocuidado e o tratamento do diabético. Assim, ressalta-se a importância da necessidade de efetivação de estratégias e programas para a redução de agravos e complicações por DM.

Acerca da atualização do conhecimento, planejamento e fortalecimento das atividades desenvolvidas pelos enfermeiros, evidenciaram-se os treinamentos, as capacitações e os encontros multidisciplinares.

Entre as dificuldades encontradas para a realização da prevenção, estão: a formação acadêmica insatisfatória; ausência de enfermeiro especialista na área; conhecimento inadequado dos enfermeiros sobre a prevenção do pé diabético; realização de anamnese e exame físico incompletos; falta de recursos materiais; fatores sociais (como falta de saneamento básico e irregularidade do abastecimento de água, o que dificulta a higienização pessoal e do lar, principalmente de usuários com úlceras extensas).

\section{DISCUSSÃO}

A prevenção do pé diabético é um tema importante de saúde pública e que vem sendo discutida em diferentes partes do mundo por diversos profissionais. Nesse contexto, a enfermagem destaca-se por promover ações educativas para conscientizar e sensibilizar a população acerca da prevenção por complicações da DM. O profissional enfermeiro está em contato direto com a comunidade, por meio de consultas e visitas domiciliares, no que lhe cabe a responsabilidade de identificação precoce, promoção, prevenção e reabilitação da saúde em função da continuidade do cuidado ${ }^{(4)}$. Em virtude disso, compreende-se que o cuidado deve ser realizado mediante a participação do usuário diabético no seu processo saúde-doença.

Quanto aos cuidados realizados pelos enfermeiros segundo os estudos, pôde-se observar o repasse de informações referentes à higienização dos pés (lavagem, corte das unhas, remoção de calos e hidratação da pele) e à utilização de calçados adequados. Entretanto, foi possível perceber a ausência de orientações importantes quanto ao exame físico diário dos pés. Nesse pensamento, ressalva-se que a avaliação rotineira dos pés é primordial na identificação precoce dos fatores de risco para o pé diabético. Além disso, o autocuidado com os pés é um fator indispensável na prevenção primária, pois pode evitar o aparecimento de ulceras e melhorar a expectativa de vida do paciente.

Quanto à avaliação dos pés, o instrumento mais utilizado pelos enfermeiros foi o Monofilamento de $10 \mathrm{~g}(5,07 \mathrm{U})$ de Semmes-Weinstein. Esse instrumento consiste em pressionar (tocar) com a ponta de um fio de nylon especial (monofilamento) algumas áreas da superfície do pé para testar sua sensibilidade a essa pressão. A incapacidade de sentir a pressão necessária para curvar o monofilamento de $10 \mathrm{~g}$ é compatível com comprometimento da sensibilidade local à pressão, ou sensibilidade $\operatorname{protetora}^{(11)}$.

Portanto, trata-se de uma tecnologia que detecta alterações, na sensação do tato e da própria percepção, de fácil manuseio e de identificação precisa. Destaca-se por ser aconselhado como teste de escolha nas avaliações por não especialistas para determinar um risco aumentado de ulceração pelas vantagens da grande sensibilidade, boa especificidade, simplicidade e o baixo custo ${ }^{(12)}$.

Contudo, a literatura recomenda, para a avaliação dos pés, que o profissional de saúde utilize pelo menos o teste com monofilamento de $10 \mathrm{~g}$ associado a outro teste e que, sempre que possível, o teste de avaliação de reflexos deve ser incluído nessa avaliação ${ }^{(13)}$.

O monofilamento de $10 \mathrm{~g}$ não é adequado para o diagnóstico mais precoce da polineuropatia, porque não detecta o acometimento das fibras finas, responsáveis pela sensibilidade dolorosa superficial e térmica, pois avalia apenas a percepção da pressão (tato) ${ }^{(11)}$. Logo, a utilização de apenas um instrumento para realizar o exame físico dos pés é visto como insuficiente na deteç̧ão precoce dos fatores de risco para o pé diabético, pois cada instrumento possui sua especificidade na identificação de alterações.

Assim, a consulta de enfermagem, como atribuição do enfermeiro na atenção básica, integrada ao exame físico dos pés, revela-se dispositivo importante na prevenção de futuras complicações. Porém, observa-se que esse profissional tem perdido a oportunidade de realizar a avaliação dos pés por vários motivos, entre os quais destacam-se: a falta de infraestrutura, desconhecimento, demanda excessiva ${ }^{(14)}$. 
Ante o exposto, reafirma-se que a avaliação dos pés dos pacientes diabéticos é imprescindível para a qualidade da assistência de enfermagem e a prevenção de complicações por ulceras, além de incentivar a prática do autocuidado e o repasse das orientações necessárias ${ }^{(15)}$. A ausência dessa prevenção pode aumentar os riscos para o surgimento de lesões.

Desse modo, é relevante enfatizar as condições de risco para o desenvolvimento de úlceras nos pés, as quais se referem às seguintes alterações: onicomicose, unha encravada, corte inadequado das unhas, edema, dermatose varizes, pulso tibial alterado, ressecamento dos pés, fissura, dedo em garra, acentuação do arco plantar, elevação do dorso plantar, proeminência metatarsiana, calos, formigamento, adormecimento e cãibras ${ }^{(16)}$. Diante disso, há que se considerar que as alterações dermatológicas locais são reflexos de entraves de ordem social e econômica enfrentados pelas pessoas diabéticas para a aquisição de calçados, palmilhas e cremes, entre outros artefatos para a prevenção de problemas nos pés ${ }^{(17)}$.

Visto isso, como medida de prevenção para o pé diabético, salienta-se o cuidado com os pés e unhas, que consiste em evitar micoses, secar a umidade entre os dedos, inspecionar sapatos e não usar calicidas, raspadores ou lâminas nos calos, que são ações igualmente importantes na prevenção primária da ulceração e amputação de membros inferiores ${ }^{(17)}$. Dentre as intervenções preconizadas para os clientes com diabetes, enfatiza-se também o uso constante de calçados apropriados, que contemplem a necessidade de cada pessoa ${ }^{(18)}$. Por esse motivo, os calçados devem apresentar: profundidade extra, que permite adaptação de palmilhas removíveis; acolchoamento interno; ausência de costuras interiores; sistema de fechamento ajustável (velcro ou cadarço); ponta quadrada e saltos de até três centímetros. Cabe citar ainda que é importante que os calçados cubram totalmente os dedos e o calcanhar e que sejam confeccionados com couro macio ou lona ${ }^{(19)}$.

Todavia, faz parte desse esforço preventivo conhecer as experiências prévias quanto ao conhecimento e comportamento que os diabéticos apresentam em relação aos cuidados com os pés. Para alcançar as metas da educação em diabetes, o paciente deve ser estimulado a desenvolver uma postura pró-ativa em relação ao seu autocuidado(20).

$\mathrm{O}$ autocuidado corresponde à forma de proteção indispensável para evitar as complicações crônicas $^{(21)}$. De forma complementar, os profissionais de enfermagem têm como desafio estimular o diabético à autonomia do cuidado, por meio de orientações completas e atualizadas quantoàs diretrizes para assistência ao pé diabético.

Destarte, observa-se que a atuação dos profissionais de saúde na orientação de autocuidado da doença envolve compreender e avaliar o indivíduo, proporcionando apoio emocional e clínico, além de conhecimentos e habilidades para alcance dos objetivos, ajudando-os a descobrir e desenvolver a autonomia para serem responsáveis pelo controle de sua doença. No entanto, os profissionais da atenção básica à saúde, na maioria das vezes, por iniciativa própria desenvolvem ações educativas voltadas aos usuários, e ressentem-se da falta de capacitação quando o tema em questão é educação em Diabetes ${ }^{(22,23)}$.

Por esse fato, além do conhecimento técnico-científico de cada área de atuação, o profissional precisa ser instrumentalizado para atuar frente às necessidades de saúde da população através de uma atuação interdisciplinar, multiprofissional e em rede, a fim de garantir o cuidado integral. A formação dos profissionais de saúde precisa evoluir com o propósito de construir um profissional capaz de conduzir seu processo de aprendizagem ao longo da vida, sendo capaz de se adaptar às mudanças, raciocinando criticamente e tomando decisões fundamentadas em sua própria avaliação(24).

Nesse aspecto, faz-se necessário que os profissionais da saúde repensem sobre suas práticas e formação acadêmica no tocante à atuação e às ações de enfermagem, procurando identificar precocemente os riscos e complicações que afetam o indivíduo com pé diabético ${ }^{(25)}$. A busca por qualificação profissional deve virar rotina entre as equipes multiprofissionais de saúde, com o propósito de conhecer a patologia, o tratamento e as formas de educação em saúde para o planejamento dos cuidados preventivos.

O enfermeiro, como parte fundamental da equipe, deve assumir a educação em saúde como seu principal foco de atuação nas ações pedagógicas, destacando que o controle e a prevenção de complicações da diabetes são possíveis por meio de programas educativos e profissionais de saúde capacitados para atuarem no processo educativo ${ }^{(26)}$.

Para evitar o aumento do número de complicações nas extremidades inferiores, programas educativos e de prevenção devem ser implementados, sendo necessário também o seu monitoramento por parte da equipe de saúde, sobretudo do enfermeiro, um dos profissionais responsáveis pela adesão do paciente ao tratamento e às ações educativas com vistas à prevenção dos agravos decorrentes do $\mathrm{DM}^{(27)}$.

Apesar das inúmeras estratégias e políticas desenvolvidas pelos serviços de saúde, as ações de prevenção não estão sendo efetivas, pois as internações por complicações da DM continuam aumentando ${ }^{(28)}$. Assim, considera-se a necessidade de formação do pensamento crítico e emancipatório dos profissionais de saúde, de forma a procurar uma comunicação aberta e interativa com os usuários, levando-os a adquirir conhecimentos e habilidades e permitindo-os fazer algo para melhorar a educação do autogerenciamento dos cuidados da doença ${ }^{(29)}$.

$\mathrm{O}$ autogerenciamento da diabetes é um objetivo universalmente considerado como fundamental nas estratégias educacionais para o controle da doença ${ }^{(30)}$. A educação em saúde para diabetes passa então a ser conceituada como um processo de problematização sobre condições de saúde e qualidade de vida, propiciando mudanças individuais, coletivas e institucionais que visam transformar a realidade. Ela é um dos mais importantes investimentos de longo prazo, já que os custos da saúde dos indivíduos com diabetes e os gastos sociais decorrentes das complicações da doença são enormes ${ }^{(31)}$. 
A respeito disso, a literatura revela que $99 \%$ dos cuidados diários necessários ao tratamento da diabetes são realizados pela pessoa com DM ou seus familiares, e o maior desafio dos profissionais de saúde consiste em estabelecer um processo efetivo de educação em saúde para promoção do desenvolvimento do autocuidado ${ }^{(32)}$.

Corroborando, identificou-se neste estudo que a abordagem educativa mais utilizada pelos enfermeiros para prevenção do pé diabético foram as atividades de educação em saúde, que se concentravam- em sensibilizar e estimular os diabéticos para modificações no estilo de vida, buscando conduzi-los para o autonomia do cuidado e enfrentamento do tratamento.

Apesar da efetivação dos programas de prevenção e a adesão às medidas profiláticas, ainda existem muitas barreiras para reduzir satisfatoriamente os fatores de risco associados ao pé diabético e suas complicações. A falta de diagnóstico ou o seu atraso, a não vigilância das situações de risco e a não implementação de medidas profiláticas estão ainda frequentemente associadas ao aparecimento de lesões graves ${ }^{(33)}$.

Para o estudo, identificou-se que as dificuldades enfrentadas pelos enfermeiros para empregar as atividades de prevenção influenciaram negativamente no planejamento das ações, na educação em saúde e na adesão ao tratamento. Revela-se ainda que os problemas na adesão ao tratamento consistem em inúmeros fatores, tais como: a baixa escolaridade, o nível socioeconômico, o déficit de conhecimento acerca da doença e obstáculos para o acesso ao serviço de saúde ${ }^{(34)}$.

Dessa forma, a partir da realidade vivenciada pela equipe de saúde frente à demanda de serviços e de escassos recursos materiais, surge a necessidade de implementar medidas que proporcionem a melhoria da assistência e prevenção de complicações da neuropatia diabética, que podem reduzir os gastos públicos e demais procedimentos cirúrgicos (desbridamentos e amputações ${ }^{(35)}$.

$\mathrm{O}$ trabalho em equipe na atenção à diabetes mellitus favorece o amadurecimento dos profissionais para compreender as necessidades reais do cliente para a conquista de sua própria saúde e a adoção de hábitos de vida saudáveis, permitindo maior eficiência e menor custo efetivo nos programas educativos. É necessário, porém, investimento na educação continuada da equipe multiprofissional e a capacitação para o autogerenciamento em doenças crônicas não transmissíveis ${ }^{(36)}$.

Para tanto, o enfermeiro e os demais profissionais do serviço de saúde devem estar dispostos a elaborar estratégias preventivas para o pé diabético que envolvam a mudança do estilo de vida e a promoção do autocuidado com os pés. Os membros da família também devem ser envolvidos nos cuidados que a doença requer ${ }^{(37,38)}$.

Nesse seguimento, faz-se necessário que os profissionais de saúde, em especial o enfermeiro, atuem no contexto educativo, uma vez que as práticas educativas também contemplam a assistência, qualquer que seja o nível de atenção à saúde. Dessa forma, através das orientações adequadas nos serviços de saúde, o cliente diabético poderá aumentar seu conhecimento e, consequentemente, melhorar sua qualidade de $\operatorname{vida}^{(39)}$.

As orientações encontradas nos estudos, visando à prevenção de complicações com o pé diabético, são efetivas no cuidado com os pés. Soma-se a isso a promoção do autocuidado, atendimento interdisciplinar, adesão ao tratamento e educação em saúde. Dessa forma, o estudo proporcionou entender que o enfermeiro tem papel fundamental enquanto profissional que atua na assistência e na educação dos usuários ${ }^{(40)}$.

Diante do exposto, acredita-se que a revisão vem para acrescentar conhecimentos acerca da temática, da importância da atuação do enfermeiro na prevenção primária e por meio da ampliação das ações básicas direcionadas aos cuidados com o diabetes e, particularmente, à prevenção de lesões nos pés. Entende-se que as orientações e cuidados realizados nos estudos podem contribuir para a prevenção de lesões nos membros inferiores, reduzindo os riscos de amputações. Há necessidade, porém, de mobilização do cuidado, com a diversificação das atividades de prevenção, que devem atender ao diabético em sua complexidade. O serviço de saúde deve oferecer infraestrutura adequada e o enfermeiro deve estar capacitado para realizar ações preventivas de qualidade.

\section{CONCLUSÃO}

Conclui-se que os enfermeiros dos artigos avaliados estão em contato diário com os pacientes diabéticos e que as ações muitas vezes estão direcionadas para atividades exclusivas de orientação quanto a doença em si, e, como consequência, o conhecimento acerca das orientações determinadas pelas diretrizes para prevenir o pé diabético torna-se insuficiente. Não obstante, o enfermeiro da atenção primária à saúde necessita conhecer mais profundamente a fisiopatologia da doença, suas comorbidades e complicações para traçar metas que conduzam à prevenção satisfatória e integral para o pé diabético, em parceria com a equipe multiprofissional, para otimizar a continuidade da assistência.

\section{REFERÊNCIAS}

1. World Health Organization. Global action plan for the prevention and control of noncommunicable diseases 2013-2020 [Internet]. Geneva: WHO; 2013 [acesso em 2016 Nov 20]. Disponível em: http:/apps.who.int/iris/ bitstream/10665/94384/1/9789241506236_eng.pdf 
2. Schmidt MI, Duncan BB, Silva GA, Menezes AM, Monteiro CA, Barreto SM, et al. Chronic noncommunicable diseases in Brazil: burden and current challenges. Lancet [Internet]. 2011 [acesso em 2016 Nov 20];377(9781):1949-61. Disponível em: http://www.thelancet.com/journals/lancet/article/PIIS0140-6736(11)60135-9/abstract

3. Ministério da Saúde (BR), Secretaria de Atenção à Saúde, Departamento de Atenção Básica. Estratégias para o cuidado da pessoa com doença crônica: Diabetes mellitus [Internet]. Brasília: Ministério da Saúde; 2013. (Cadernos de Atenção Básica, $\mathrm{n}^{\circ}$ 36) [acesso em 2016 Maio 10]. Disponível em: http://189.28.128.100/dab/docs/portaldab/publicacoes/caderno_36.pdf

4. Santos GILSM, Capirunga JBM, Almeida OSC. Pé diabético: condutas do enfermeiro. Rev Enferm Contemporânea [Internet]. 2013 [acesso em 2016 Jun 15];2(2):225-41. Disponível em: https://www5.bahiana.edu.br/index.php/ enfermagem/article/view/303

5. Fernandes AFC, Galvão CM. Métodos de revisão: não podemos banalizar. Rev Rene [Internet]. 2013 [acesso em 2016 Jun 10];4(1):1-2. Disponível em: http://www.redalyc.org/pdf/3240/324027985001_2.pdf.

6. Mendes KDS, Silveira RCCP, Galvão CM. Revisão integrativa: método de pesquisa para a incorporação de evidências na saúde e na enfermagem. Texto \& Contexto Enferm [Internet]. 2008 [acesso em 2016 Jun 15];17(4):758-64. Disponível em: http://www.scielo.br/scielo.php?script=sci_arttext\&pid=S0104-07072008000400018.

7. Cubas MR, Santos OM, Retzlaff EMA, Telma HLC, Andrade IPS, Moser ADL, et al. Pé diabético: orientações e conhecimento sobre cuidados preventivos. Fisioter Mov [Internet]. 2013 [acesso em 2016 Maio 20];6(3):647-55. Disponível em: http://www.scielo.br/pdf/fm/v26n3/a19v26n3.pdf

8. Aalaa MM, Tabatabaei Malazy O, Sanjari M, Peimani M, Mohajeri-Tehran MR. Nurses' role in diabetic foot prevention and care: a review. J Diabetes Metab Disord [Internet]. 2012 [acesso em 2016 mai 20];11(24):1-6. Disponível em: http:// www.ncbi.nlm.nih.gov/pmc/articles/PMC3598173/

9. Policarpo NS, Moura JRA, Melo EB Júnior, Almeida PC, Macedo SF, Silva ARV. Conhecimento, atitudes e práticas de medidas preventivas sobre pé diabético. Rev Gaúcha Enferm [Internet]. 2014 [acesso em 2016 Maio 20];35(3):36-42. Disponível em: http://www.scielo.br/pdf/rgenf/v35n3/pt_1983-1447-rgenf-35-03-00036.pdf

10. Varaei S. Education and implementing evidence-based nursing practice for diabete cpatients. Iran J NursMidwifery Res [Internet]. 2013 [acesso em 2016 Maio 20]; 18(3):251-7. Disponível em: http://www.ncbi.nlm.nih.gov/pmc/articles/ PMC3748547/

11. Caifa JS, Castro AA, Fidelis C, Santos VP, Silva ES, Sitrangulo JR, et al. Atenção integral ao portador de Pé Diabético. J Vasc Bras [Internet]. 2011 [acesso em 2016 Jul 10]; 10(4 Supl 2):2-32. Disponível em:_http://www.scielo.br/pdf/jvb/ v10n4s2/a01v10n4s2.pdf

12. Donoso MTV, Rosa EG, Borges EL. Perfil dos pacientes com pé diabético de um serviço público de saúde. Rev Enferm UFPE. 2013;7(7):4740-6.

13. Taddeo PS, Gomes KWL, Caprara A, Gomes AMA, Oliveira GC, Moreira TMM. Acesso, prática educativa e empoderamento de pacientes com doenças crônicas. Ciênc Saúde Coletiva [Internet]. 2012 [acesso em 2016 Jul 10];17(11):2923-30. Disponível em: http://www.scielo.br/pdf/csc/v17n11/v17n11a08.pdf

14. Amaral AS, Tavares DMS. Cuidados com os pés: conhecimento entre pessoas com diabetes mellitus. Rev Eletrônica Enferm [Internet]. 2009 [acesso em 2016 Nov 11]; 11(4):801-10. Disponível em: https://www.fen.ufg.br/fen_revista/v11/ n4/pdf/v11n4a05.pdf

15. American Diabetes Association. Standars of Medical Care in Diabetes. Diabetes Care [Internet]. 2013 [acesso em 2016 Nov 14];36(Suppl 1):11-66. Disponível em: https://www.ncbi.nlm.nih.gov/pmc/articles/PMC3537269/pdf/S11.pdf

16. Pereira FGF, Diógenes MAR, Freire DF, Meneses MS, Xavier ATF, Ataíde MBC. Abordagem clínica de enfermagem na prevenção do pé diabético. Rev Bras Promoç Saúde [Internet]. 2013 [acesso em 2016 Jun 21];26(4):498-504. Disponível em: http://www.unifor.br/images/pdfs/rbps/2013.4_artigo6.pdf

17. Ochoa-Vigo K, Pace AE. Pé diabético: estratégias para prevenção. Acta Paul Enferm [Internet] 2005 [acesso em 2016 Jul 25];18(1):100-9. Disponível em: http://www.scielo.br/pdf/ape/v18n1/a14v18n1.pdf

18. Silva CL, Moleta C, Severo E, Gaspar MDR, Cavalheiro MA. Características de lesões de pé diabético e suas complicações. Rev Rene [Internet]. 2012 [acesso em 2016 Maio 20]; 13(2):445-53. Disponível em:_http://www.redalyc. org/pdf/3240/324027981021.pdf

19. Péres DS, Santos MA, Zanetti ML, Ferronato AA. Difficulties of diabetic patients in the illness control: feelings and behaviors. Rev Latinoam Enferm [Internet]. 2007 [acesso em 2016 Nov 22];15(6):1105-12. Disponível em: http://www. scielo.br/scielo.php?script=sci_arttext\&pid=S0104-11692007000600008 
20. Faber DC, Faber JS. Office-based screening, prevention, and management of diabetic foot disorders. Prim Care [Internet]. 2007 [acesso em 2016 Jun 21];34(4):873-85. Disponível em: http://www.ncbi.nlm.nih.gov/pubmed/18061821

21. Torres HC, Franco LJ, Stradioto MA, Hortale VA, Schall VT. Avaliação estratégica de educação em grupo e individual no programa educativo em diabetes. Rev Saúde Pública [Internet]. 2010 [acesso em 2016 Jun 21];43(2):291-8. Disponível em: http://www.scielo.br/scielo.php?script=sci_arttext\&pid=S0034-89102009000200010.

22. Torres HC, Monteiro MRP. Educação em saúde sobre doenças crônicas não-transmissíveis no Programa Saúde da Família em Belo Horizonte/MG. REME Rev Min Enferm. 2006;10(4):402-6.

23. Rocha RM, Zanetti ML, Santos MA. Comportamento e conhecimento: fundamentos para prevenção do pé diabético. Acta Paul Enferm [Internet]. 2009 [acesso em 2016 Nov 20]; 22(1):17-23. Disponível em: http://www.scielo.br/pdf/ape/v22n1/ a03v22n1.pdf

24. Couto TA, Santana VSS, Santos AR, Santos RMM. Educação em saúde, prevenção e cuidado ao pé diabético: um relato de experiência. Rev Baiana Saúde Pública [Internet]. 2014 [acesso em 2016 jun 25];38(3):760-8. Disponível em: http://inseer. ibict.br/rbsp/index.php/rbsp/article/viewFile/685/pdf_590

25. Zanetti ML, Otero LM, Freitas MCF, Santos MA, Guimarães FPM, Couri CEB, et al. Atendimento ao paciente diabético utilizando o protocolo staged diabetes management: relatos de experiência. Rev Bras Promoç Saúde [Internet]. 2006 [acesso em 2016 Jun 25]; 19(4):253-60. Disponível em: http://www.redalyc.org/articulo.oa?id=40819411

26. Santos CV Filho, Rodrigues WHC, Santos RB. Papeis de autocuidado: subsídios para enfermagem diante das reações emocionais dos portadores de diabetes mellitus. Esc Anna Nery Rev Enferm. [Internet]. 2008 [acesso em 2016 Jun 25];12(1):125-9. Disponível em: http://www.scielo.br/pdf/ean/v12n1/v12n1a19

27. Sociedade Brasileira de Diabetes. Detecção e tratamento das complicações crônicas do Diabetes Mellitus [Internet]. 2008 [acesso em 2016 Jun 20]. Disponível em: http://www.diabetes.org.br/educacao/ compcrondoc.php.

28. Carácio FCC, Conterno LO, Oliveira MAC, Oliveira ACH, Marin MJS, Braccialli LAD. A experiência de uma instituição pública na formação do profissional de saúde para atuação em atenção primária. Ciênc Saúde Coletiva [Internet]. 2014 [acesso em 2016 Jun 21];19(7):2133-42. Disponível em: http://www.scielo.br/scielo.php?pid=S1413$81232014000702133 \&$ script $=$ sci_abstract\&tlng $=$ pt

29. Arruda C, Silva DMCGV. Acolhimento e vínculo na humanização do cuidado de enfermagem às pessoas com diabetes mellitus. Rev Bras Enferm [Internet]. 2012 [acesso em 2016 Jun 25];65(5):758-66. Disponível em:_http://www.scielo.br/ pdf/reben/v65n5/07.pdf

30. Harris SB, Yale JF, Berard L, Stewart J, Abbaszadeh B, Webster-Bogaert S, et al. Does a Patient-Managed Insulin Intensification Strategy With Insulin Glargine and Insulin Glulisine Provide Similar Glycemic Control as a PhysicianManaged Strategy? Diabetes Care [Internet]. 2014 [acesso em 2016 Dez 15];37(3):604-10. Disponível em: https://www. ncbi.nlm.nih.gov/pubmed/24170757.

31. Ministério da Saúde (BR), Secretaria de Atenção à Saúde, Departamento de Atenção Básica. Diabetes mellitus [Internet]. Brasília: Ministério da Saúde; 2016. (Cadernos de Atenção Básica, no 16) [acesso em 2016 Dez 15]. Disponível em: http://189.28.128.100/dab/docs/publicacoes/cadernos_ab/abcad16.pdf

32. Torres HC, Amaral MA, Amorim MM, Cyrino AP, Bodstein R. Capacitação de profissionais da atenção primária à saúde para educação em Diabetes Mellitus. Acta Paul Enferm [Internet]. 2010 [acesso em 2016 Jun 25];23(6):751-6. Disponível em: http://www.scielo.br/pdf/ape/v23n6/06.pdf

33. Sociedade Brasileira de Diabetes. Diretrizes da Sociedade Brasileira de Diabetes [Internet]. $3^{\text {a }}$ ed. Itapevi: A. Araujo Silva Farmacêutica; 2009 [acesso em 2016 jun 20]. Disponível em: http://dms.ufpel.edu.br/ares/bitstream/ handle/123456789/270/11\%20\%20\%202009\%20diretrizes\%20diabete.pdf?sequence=1

34. Melo EM, Teles MS, Teles RS, Barbosa IV, Studart RMB, Oliveira MM. Avaliação dos fatores interferentes na adesão ao tratamento do cliente portador de pé diabético. Rev. Enf. Ref. [Internet]. 2011. [acesso em 2016 jun 25];3(5):33-47. Disponível em: http://www.scielo.mec.pt/pdf/ref/vserIIIn5/serIIIn5a04.pdf

35. Santos ICRV, Nunes ENS, Melo CA, Farias DG. Amputações por pé diabético e fatores sociais: implicações para cuidados preventivos de enfermagem. Rev Rene [Internet]. 2011 [acesso em 2016 Jun 20];12(4):684-91. Disponível em: http://www. repositorio.ufc.br/bitstream/riufc/12688/1/2011_art_icrvsantos.pdf

36. Silva CAM, Pereira DS, Almeida DSC, Venâncio MIL. Pé diabético e avaliação do risco de ulceração. Rev Enferm Ref [Internet]. 2014 [acesso em 2016 Jun 25];4(1):153-61. Disponível em: http://www.scielo.mec.pt/pdf/ref/vserIVn1/ serIVn1a17.pdf 
37. Dantas DV, Costa JL, Dantas RAN, Torres GV. Atuação do enfermeiro na prevenção do pé diabético e suas complicações: revisão de literatura. Carpe Diem [Internet]. 2013 [acesso em 2016 Jun 23];11(11):1-14. Disponível em: https://periodicos. unifacex.com.br/Revista/article/view/359/113

38. Cisneros LL, Gonçalves LAO. Educação terapêutica para diabéticos: os cuidados com os pés na realidade de pacientes e familiares. Cienc Saude Coletiva [Internet]. 2011 [acesso em 2016 Jun 20];16 Supl 1:1505-14. Disponível em: http://www. scielo.br/scielo.php?script=sci_arttext\&pid=S1413-81232011000700086

39. Morais GFC, Soares MJGO, Costa MML, Santos IBC. Conhecimento e práticas dos diabéticos acerca das medidas preventivas para lesões de membros inferiores. Rev Baiana Enferm [Internet]. 2009 [acesso em 2017 Jan 05];33(3):36171. Disponível em: http://inseer.ibict.br/rbsp/index.php/rbsp/article/viewFile/219/pdf_48

40. Tavares DMS, Dias FA, Araújo LR, Pereira GA. Perfil de clientes submetidos a amputações relacionadas ao diabetes mellitus. Rev Bras Enferm [Internet]. 2009 [acesso em 2017 Jan 06];62(6):825-30. Disponível em: http://www.scielo.br/ pdf/reben/v62n6/a04v62n6.pdf.

\section{Endereço para correspondência:}

Ana Roberta Vilarouca da Silva

Universidade Federal do Piauí - UFPI

Campus Universitário Ministro Petrônio Portella

Bairro: Ininga

CEP 64049-550 - Teresina - PI - Brasil

E-mail: robertavilarouca@yahoo.com.br 